\title{
Corrosion Effects on the I-V Characteristics of Electrically Conducting Cables
}

\author{
*ABBEY, T M; OBONG, H P \\ Department of Physics, University of Port Harcourt, Nigeria
}

\begin{abstract}
Experimental analysis on the effects of atmospheric Pollution and environmental degradation on the electrical properties of un-protected high tension cables, using copper and Aluminum wires of various diameters as case study, has been advanced. The analysis of the various data obtained in the course of the experiment, using the Aitken Interpolation model, shows that exposure of electrical conducting cables to humid, highly polluted and toxic atmosphere, decreases the I - V (current - voltage) characteristic as well as the conductivity of the cable due to increase in the amount of degradation and weight losses. Further analysis indicates that thicker cables suffer less degradation and power loss compare to thinner ones. @JASEM
\end{abstract}

In recent times, environment and its associated problems have been a major concern to different categories of persons and interest groups. Chiefly, because environmental pollutions, which might occur either as a result of the discharge of dangerous chemical into the atmosphere as effluent or the deposition of organic matter into the water bodies and soil, have resulted in server economics and human lost to both the developing and industrial worlds. For example a satellite Memorial report published in 1978 indicates that corrosion cost United States 8.2 billion dollars, which is a $4.9 \%$ of her gross national income, on equipment and facility maintenance (Ebiogwu, 1992, Johnson, 1992 and Brikby et al, 1999).

Therefore, due to the increasing effects of industrial pollution on the environment [Dawson and Turner (1988), Nelson and Stile (1988) and Abbey et al (2003)], several researchers have devoted a great deal of attentions to the study of the impact of environmental degradations on several forms of human activities. Abbey et al (1997) for example, presented a mathematical model on the effect of pollutant oscillations on oil spreading on the sea as away of simulating the hazard that might occur due to an asymmetrical, night-time, oil spillage on the sea. On the other hand, Bansal (1976) considered the effect of industrial pollutants on natural streams and big blue rivers when the source is instantaneous. Amens (1988), Cleary (1976), Mckelliget et al (1982) and Wenk and Fochtman (1972) have in their various ways presented models to simulate the effects of pollutants transport in rivers. In a similar development, Owate et al (1999) shows that the release of hydrocarbon substances into the atmosphere, which resulted in the formation of acid rain, in-hibited the electrical properties of some exposed appliances. Similarly, Chinemeran (1989) investigated the effect of environmental degradation on silicon semiconductor wafers and observed that, changes in the environmental conditions resulted in the variation in the number of charge carriers present in the semiconductor. Others such as, Ezeh (1992), Akpan et al (1992), Triki et al (1992), Allen and Hilbert (1992), Cookey and Okongwu (1992), Owate (1992) and Owate and Akpata (1998) have examined respectively the impact of oil field corrosion on the resistance of Alumina and Aluminium nitride materials.

In all the above studies, none have examined the effect of oil field industrial pollution and acid rain resulting from gas flaring on the electrical properties of high tension cables. This present study, following Owate et al (1999) is intended to addressed this problem. To embark on the investigations, the required corrosive medium shall be prepared from polluted water obtained from a highly polluted oil field environment. Electrical cables of known diameter and resistivity will be immersed into the medium for a couple of weeks and thereafter a current - voltage measurement will be carried out.

The following format is adopted in the report of the experimental analysis. Section 2 deals with the materials and methods which include the I-V measurement procedure, while section 3 present the results and their analysis Section 4 on the other hand presents the quantitative discussion of the results.

\section{MATERIALS AND METHODS}

Sample Collection and Preparation: Metals, particularly copper, are mostly used as electrical cables due to their unique electrical properties. Therefore, coils of copper and Aluminum wires with fairly uniform cross-sections of diameters 8 and $10 \mathrm{~mm}$ respectively were obtained from Cables and industrial Supplies Limited. The Cables were striped of its protective coating and sectioned into a length of $100 \mathrm{~cm}$ each and thereafter washed in warm water and subsequently rinsed in de-ionized water and in ethanol solution in order to get rid of

* Corresponding Author (E-mail: abbeytams@yahoo.co.uk 
all form of impurities that could contribute to the mass of the sample. The samples were later airdried for twenty minutes after the cleansing processes outlined above following the method in Owate et al (1999).

The expected corrosive media were prepared by adding 1 molar solution of concentrated sulphuric acid $\left(\mathrm{H}_{2} \mathrm{SO}_{4}\right)$ and 2 molar solutions of concentrated hydrochloric acid (HCL) to every 1 litre $\left(1000 \mathrm{~cm}^{3}\right)$ of effluent water obtained from a highly toxic and polluted oil field environment in the Niger Delta of the Nigeria Nation State. The final solutions were later exposed to the atmosphere for a period of two months (60 days) in order to enhance the absorption of oxygen, carbon-dioxide and other trace of gaseous substances in the atmosphere.

At the expiration of the 60days of exposure, a chemical analysis was carried out on the exposed solutions using the atomic mass absorption photospectrometer. The analysis reveals the presence of the following substances, in the exposed corrosive medium.
Table 1. Provide caption

\begin{tabular}{ll}
\hline Substance & Concentration \\
\hline Iron (total) & 0.70 \\
$\mathrm{SO}_{4}^{2-}$ & 1.70 \\
$\mathrm{NO}^{3-}$ & 3.70 \\
$\mathrm{Ca}^{2+}$ & 22.11 \\
$\mathrm{Mg}^{2+}$ & 1057 \\
$\mathrm{CO}_{3}^{2-}$ & 0.00 \\
$\mathrm{Bi}-$ Carbonate & 499.89 \\
Salinity & 8267.74 \\
TDS (g/c) & 6.77 \\
Conductivity & 13.01 \\
Turbidity (FTU & 22.0 \\
pH & 4.0 \\
\hline
\end{tabular}

Finally the $100 \mathrm{~cm}$ length pieces of wire (samples) were weighed, using electro-sensitive balance and the mass $(\mathrm{m})$ before exposure to the corrosive medium determined. The weighed samples were then immersed in the prepared corrosive solution (medium), contained in a bath, for a period ranging from 30days to 180days. Some of the immersed samples (soaked cables) were subsequently transferred, after the completion of their immersion period, into another bath of pure water and allowed to re-soaked for 30minutes. This is done possibly, to quench or reduce the burning effect of the acidic medium on the wire.

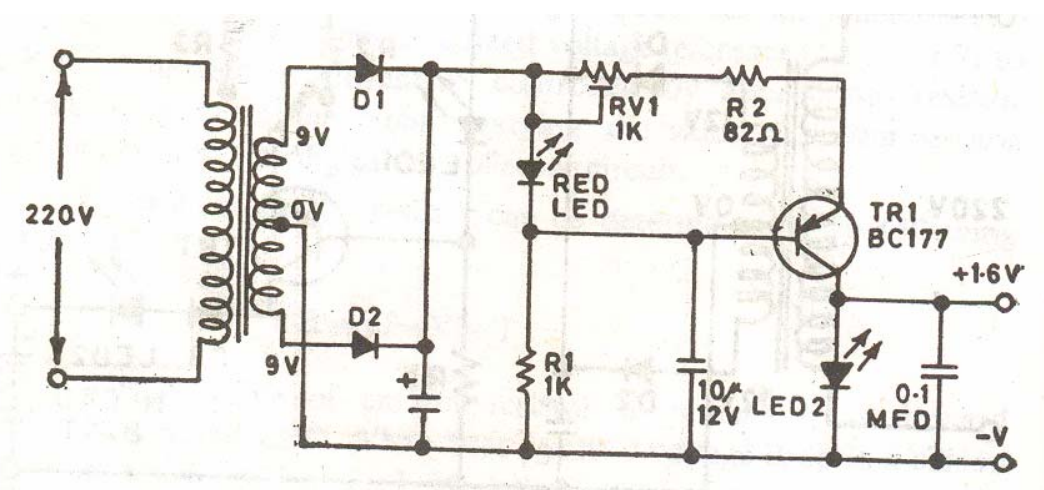

Fig. 1: The Electrical Circuit for the I-V Measurement.

The I.V. Measurement: The three categories of samples, namely, the controlled samples (unsoaked), the samples immersed in the corrosive medium (the soaked) and those that were reimmersed in water for 30minutes, (the re-soak) were all subjected to the current-voltage measurement using the electrical circuit arrangement shown in Figure 1. The pd, that is the voltage drop (v) across each piece of sample was pre-set on the voltmeter $(\mathrm{Vm})$ using the rheostat (Rh) and the corresponding current (I) registered on the Ammeter (A) recorded. The results of the measurement are as presented on Tables (1) for both the controlled, the soaked and the re-soaked samples respectively.

The D-Factor (Power Loss) Measurement: The electrical conductivity of the samples was measured as a function of frequency, using the frequency response analyzer and the impedance meter. The 
Schlumberger Frequency Response Model 18900 and the Solariton impedance Analyser Model 176, $10 \mathrm{H}_{3}-1 \mathrm{M}_{3}$ were used and the power loss or the DFactor calculated from the data obtained. The results are as stated on Table 1 .

Table 1: D-Factor Measurement

\begin{tabular}{ccc}
\hline $\begin{array}{l}\text { Frequency } \\
\left(\mathrm{KH}_{3}\right)\end{array}$ & $\begin{array}{l}8 \mathrm{~mm} \\
\text { Diameter } \\
\left(\times 10^{-3}\right)\end{array}$ & $\begin{array}{l}10 \mathrm{~mm} \\
\text { Diameter } \\
(\mathrm{x} \mathrm{10})\end{array}$ \\
\hline 0.05 & 0.45 & 41.00 \\
10.00 & 3.00 & 65.00 \\
20.00 & 9.00 & 82.00 \\
50.00 & 56.00 & 818.00 \\
70.00 & 82.00 & 1204.00 \\
\hline 90.00 & 889.00 & 1701.00 \\
\hline
\end{tabular}

\section{RESULTS AND ANALYSIS}

Calculation of Weight Loss and Corrosion Rate:

Both the soaked and the re-soaked samples were re- weighed and their masses and weight losses determined, by taking the different in mass between the soaked and the un-soaked or controlled samples. The corrosion rate in mass per year (MPY) is calculated using the relation

Corrosion rate $(\mathrm{MPY})=\frac{534}{\rho_{o} A}\left(\frac{\Delta W}{\Delta t}\right)$

as in Owate et al (1999), where $\rho_{\mathrm{o}}$ is the density of the exposed sample, $\Delta t$, the time of immersion or exposure in the corrosive medium and $\mathrm{A}=\frac{\pi d^{2}}{4}$ is the cross-sectional area of the wire while $d$ is its diameter. The result for the mass $(\mathrm{m})$, weight loss $(\Delta \mathrm{W})$ and corrosion rate $(\gamma)$ for the 8 and $10 \mathrm{~mm}$ samples are as shown on Table 2

Table 2: Mass Loss, Corrosion Rates and Electrical Conductivity

\begin{tabular}{|c|c|c|c|c|c|c|c|c|c|c|}
\hline \multirow{2}{*}{$\begin{array}{l}\text { Period of } \\
\text { exposure } \\
\text { in days }\end{array}$} & \multicolumn{5}{|c|}{ FOR 8mm SAMPLE } & \multicolumn{5}{|c|}{ FOR 10mm DIAMETER } \\
\hline & 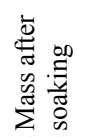 & 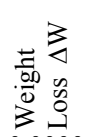 & 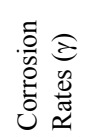 & 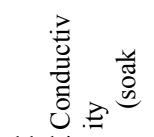 & 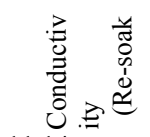 & 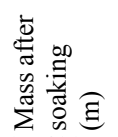 & 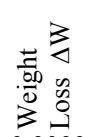 & 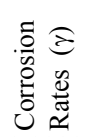 & 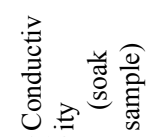 & 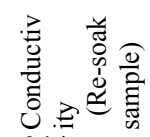 \\
\hline 0.0 & .4040 & 0.0000 & 0.0000 & 11.14 & 11.14 & 0.5483 & 0.0000 & 0.0000 & 6.14 & 6.14 \\
\hline 30.0 & 0.3946 & 0.0094 & 0.8099 & 9.20 & 11.02 & 0.5432 & 0.0051 & 0.4394 & 5.64 & 5.86 \\
\hline 60.0 & 0.3808 & 0.0232 & 0.9994 & 8.45 & 9.22 & 0.5379 & 0.0104 & 0.4480 & 5.00 & 5.25 \\
\hline 90.0 & 0.3526 & 0.0514 & 1.4761 & 7.07 & 8.17 & 0.5010 & 0.0473 & 1.3583 & 4.44 & 4.61 \\
\hline 120.0 & 0.3241 & 0.0799 & 1.7210 & 5.91 & 6.99 & 0.4797 & 0.0686 & 1.4777 & 3.67 & 4.06 \\
\hline 150.0 & 0.3037 & 0.1007 & 1.7352 & 5.05 & 6.19 & 0.4540 & 0.0943 & 1.6250 & 3.22 & 3.67 \\
\hline \multirow[t]{2}{*}{180.0} & 0.2828 & 0.1212 & 1.7404 & 3.98 & 5.67 & 0.4240 & 0.1196 & 1.7173 & 2.67 & 3.00 \\
\hline & & & & X $10^{6}$ & X $10^{6}$ & & & & X $10^{6}$ & X $10^{6}$ \\
\hline
\end{tabular}

The Interpolation (Trend) Analysis: To predict the trend of the degradation of the electrical properties of the cables under the influence of hazardous medium, an interpolation analysis is carried out on the data presented on Table 3. The Aitken Lagrangian interpolation technique, which has been widely used, elsewhere following Jain et al (2004) is adopted to fit the trend for the weight loss, corrosion rate and the electrical conductivity as a function of time of exposure in the acidic medium. The analysis shows that the data on Table 3 can be interpolated using a relation of the form:-

$F(\tau)=\sum_{j=O}^{N} I_{j}(\tau) Q(\tau)$

(2)

where,

$$
I_{j}(\tau)=\frac{\prod_{k=0}^{N}\left(\tau-\tau_{k}\right)}{\left(\tau-\tau_{j}\right) \prod_{k=0}^{N}\left(\tau_{j}-\tau_{k}\right)}
$$

while $\mathrm{N}$ is the number of entries on the table, $\tau_{j}$ the jth entry on the table, $Q(\tau)$ is the measured value corresponding to the $\tau_{j}$ entry on the Table and $F(\tau)$ is the desired interpolating function that best fit the experimental data. In this study, $F(\tau)$ represents the weight loss $\Delta \mathrm{W}$ or the corrosion rate $(\gamma)$ or the electrical conductivity $(\delta$ ) of the cable and $\tau$ is the non-dimensional time of exposure of the cable to the corrosive environment. Following equations (2) and (3) the data on Table 3 have the following trend 


$$
F_{i}(\tau) \cong \sum_{K=O}^{6} a_{k} \tau^{6}
$$

Table 4 for the corrosion rate $(\gamma)$ and electrical conductivity $(\delta)$ respectively.

where the constants: $a_{k}, k=0,1,2 \ldots 6$ for the interpolating functions $\mathrm{F}_{\mathrm{i}}(\tau)$ are as presented on

Table 3: The Constants Associated with the Interpolating Functions

\begin{tabular}{|c|c|c|c|c|c|c|c|}
\hline \multirow{2}{*}{$\mathrm{F}(\tau)$} & \multicolumn{7}{|c|}{ THE CONSTANTS } \\
\hline & $\mathrm{a}_{\mathrm{o}}$ & $\mathrm{a}_{1}$ & $a_{2}$ & $a_{3}$ & $\mathrm{a}_{4}$ & $a_{5}$ & $\mathrm{a}_{6}$ \\
\hline$\gamma_{8(\mathrm{~mm})}$ & 0.000 & 2.543 & -3.084 & 1.778 & -0.487 & 0.063 & -0.003 \\
\hline$\gamma_{10(\mathrm{~mm})}$ & 0.000 & 4.464 & -7.813 & 5.390 & -1.644 & 0.233 & -0.013 \\
\hline$\delta_{8(\mathrm{~mm})}$ & 11.140 & -115.500 & 5.540 & -3.110 & 0.830 & -0.110 & 0.010 \\
\hline$\delta_{10(\mathrm{~mm})}$ & 6.140 & 0.560 & -2.150 & 1.520 & -0.500 & 0.080 & -0.010 \\
\hline
\end{tabular}

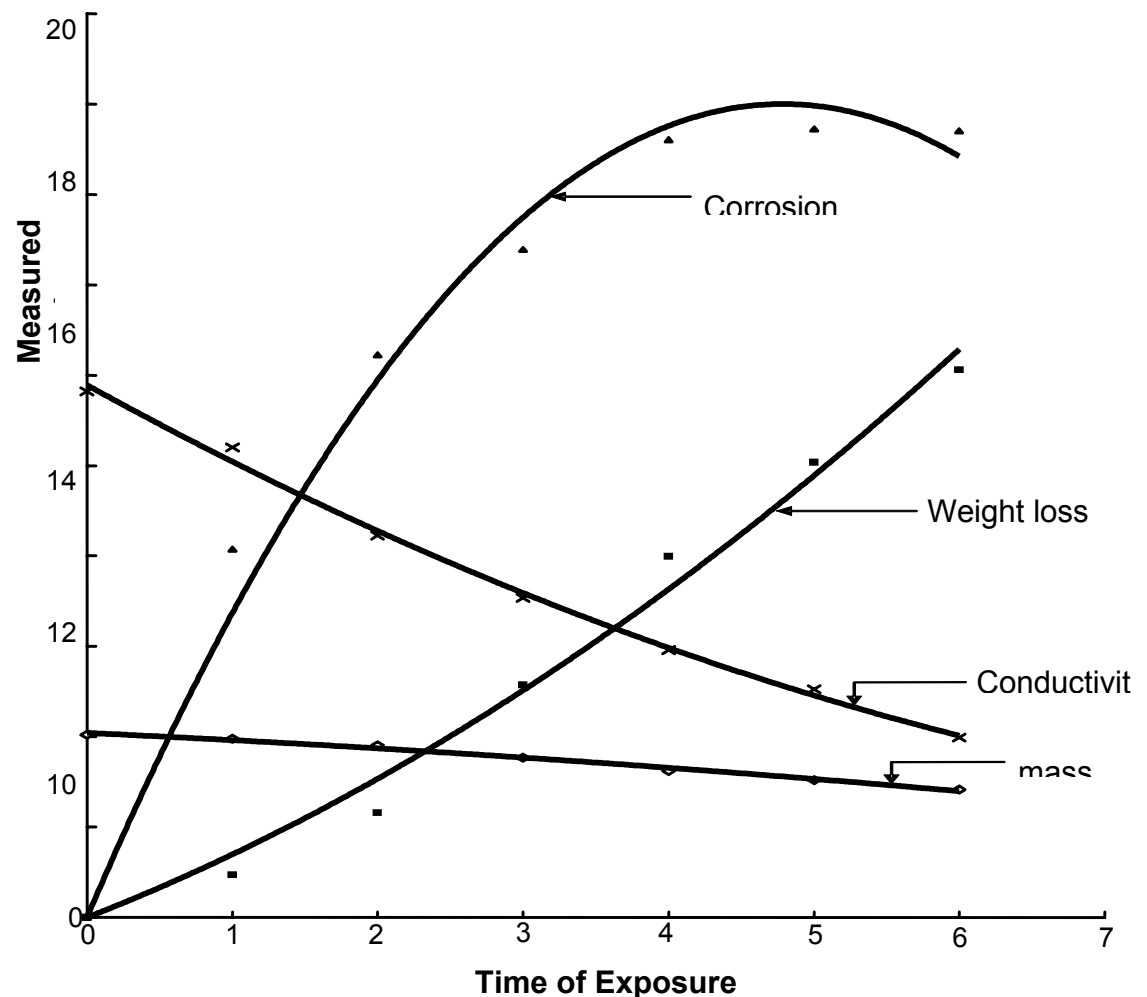

Fig. 2: Variations of measured value with time for the $8 \mathrm{~mm}$ cable. 


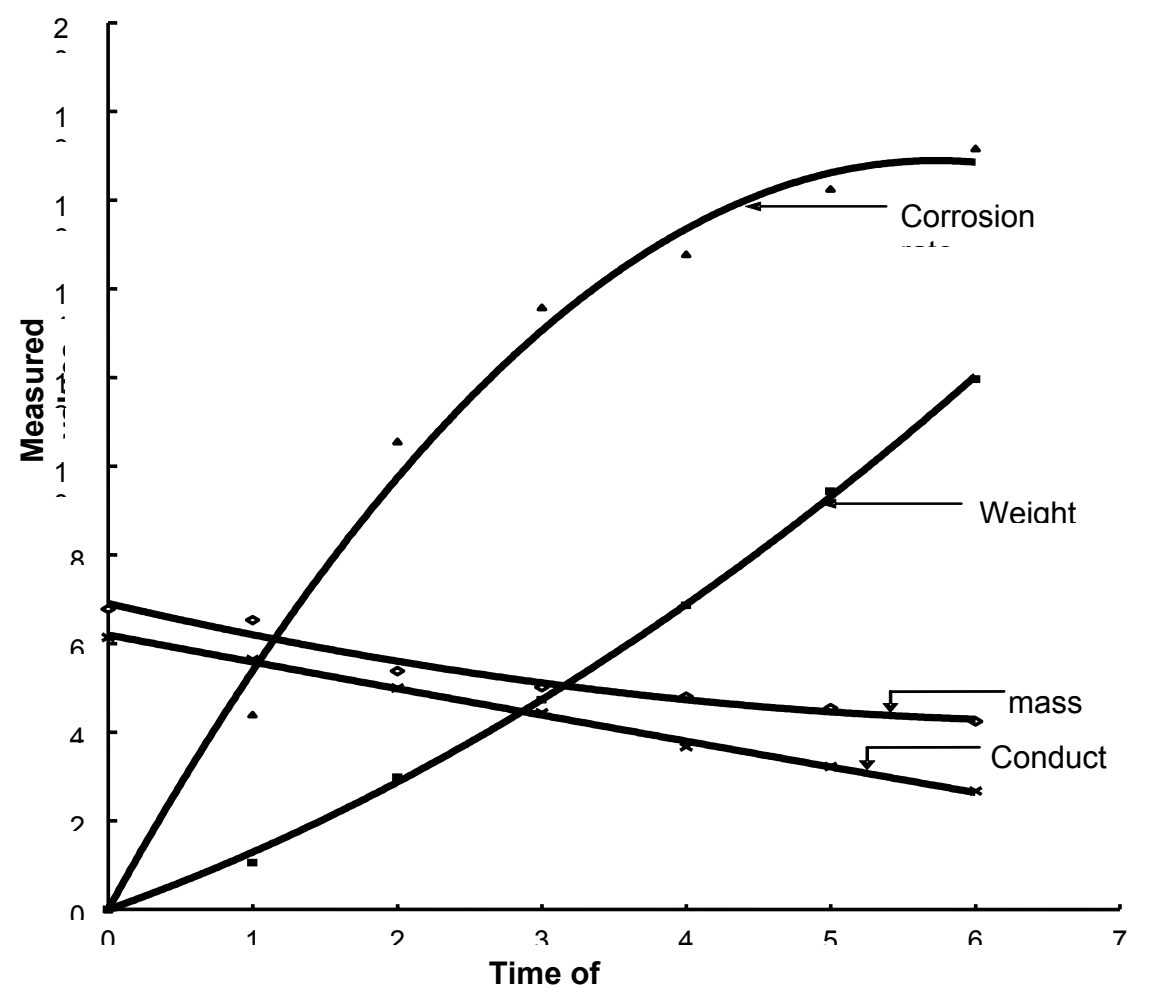

Fig. 3: Variations of measured value with time for the $10 \mathrm{~mm}$ cable.

\section{DISCUSSION}

The main thrust of this study was to investigate experimentally the effect of environmental unfriendly atmosphere on the electrical properties of high tension cables. To this end, Copper and Aluminum wires of various sizes were exposed to hazardous medium, which was prepared by a mixture of 1 mole of sulphuric acid $\left(\mathrm{H}_{2} \mathrm{SO}_{4}\right)$ and 2 moles of hydrochoric acid. (HCL) with 1litre $\left(1000 \mathrm{~cm}^{3}\right)$ of effluent water obtained from Obagi, a highly polluted oil field environment, for a period of 30,60, 90,120, 150 and 180 days respectively.

The I-V, measurements presented on Tables 1 indicate that the current (I) of the samples decreases with increase in the exposure time. Table 3 and figures 2 and 3 revealed that the mass, weight loss as well as the corrosion rate increase as the duration of exposure of the sample in the corrosive medium increases. However, the electrical conductivities are observed to exhibit opposite trend as these decrease with increase in the number of days of exposure. The investigation further reveals that although the cables experience a decrease in the I-V characteristic, but its normal Ohmic's behaviour, which is usually associated with metallic conductors still maintained. That is, the exposed cables were observed, during the I-V analysis to exhibit the normal linearity variation pattern typical ABBEY, T M; OBONG, $H P$ of electrical conducting wires. A regression analysis gives the linearity relation as $\mathrm{I}=\mathrm{gv}+\mathrm{C}$, where $\mathrm{g}$ is the conductance of the cable and $\mathrm{C}$ is a constant, which in actual case should be zero, but with the effect of the hazardous medium. $\mathrm{C}$ is observe not to be equal to zero (i.e. $C \neq 0$ ) in this case.

As observed in the case of Dawson and Turner (1988), Nelson and Stile (1988), Darry and Spear (1992) and Brikby et al (1999). The gradual and systematic eating away of the cable surface results in mechanical and electrical damaged. The power loss or D-factor analysis, indicated on Table (2) shows that the corroded cables exhibited high magnitude of power lose as a result of high current leakage due to the effect of skin friction created on the wire during the corrosion process. Consequently, the electrical conductivity and conductance of the exposed cables as shown in figures (2), (3) and (4) decreases with increase in the rate of corrosion.

This phenomenon was observed for both the soaked and the Re-soaked samples. However, the re-soaked samples were observed to exhibit a higher trend of electrical properties than the completely soaked ones. This difference is attributed to the formation of thin oxide layer on the 
cable surface during the quenching process, thereby increasing the thickness of the cable and which

subsequently affects its conductivity.

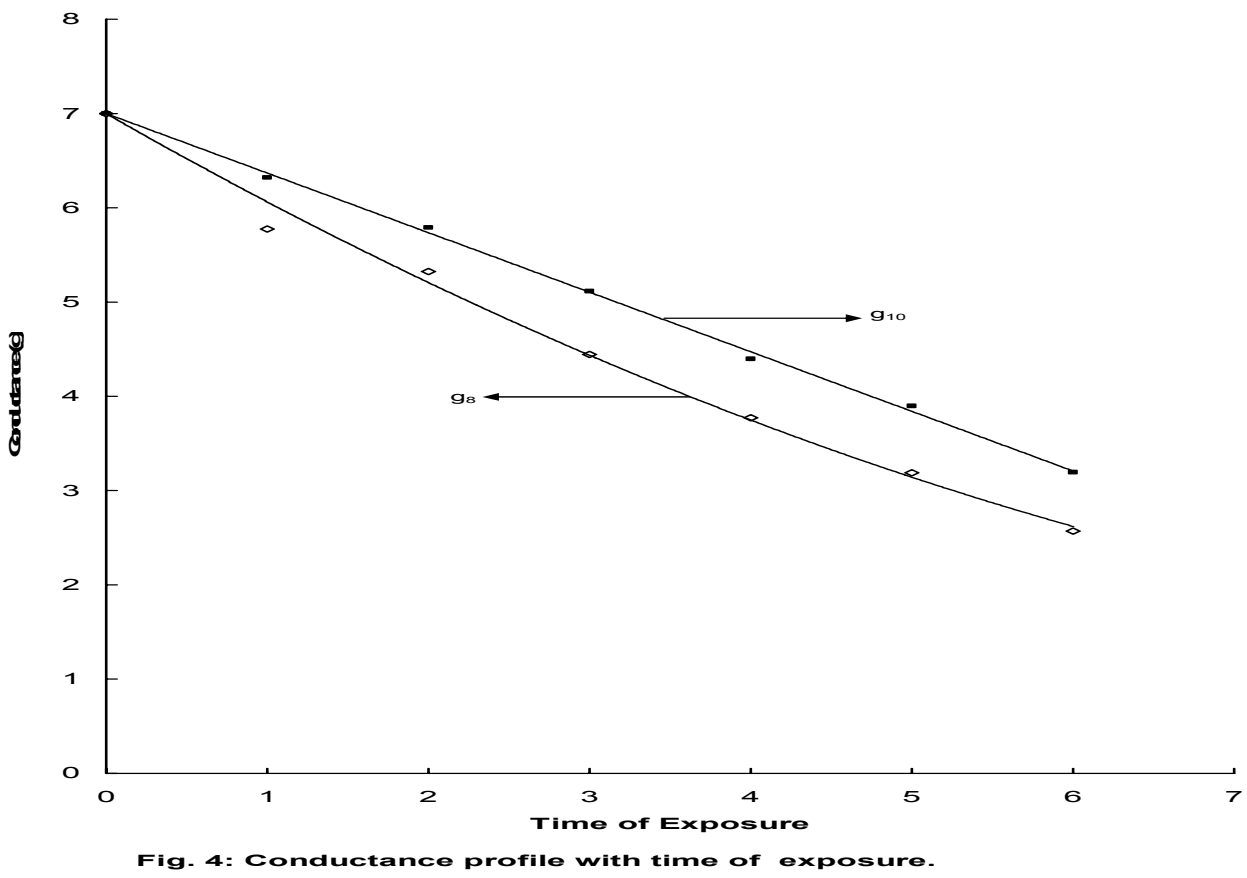

Secondly, the decrease in the conductivities of the samples can easily be explained base on the thermodynamics and reaction kinetic principles of metals in contact with corrosive systems. That is, the mechanism of metallic corrosion requires that the metal in question be oxide first in the presence of another system that has ability to accept electrons released from metal surface. It follows that if a metal is less active than the system in which it is in contact, there will be no transfer of electrons and as such the metal will not corrode. The D-factor analysis shows that the hazardous medium used for the investigation was a noble one, that is, a system or medium that is less active compared to the copper wire. This enable the transfer of energy to the solution, thereby facilitating the corrosion process and subsequently mass and weight lose observed.

Generally, the interpolation analysis, shown in figures (2), (3) and (4) are in good agreement with the experimental data. The trend show that for a prolong period of exposure, the cables experience passivation, and this leads to the stabilizing and set back effects observed in the experimental results.

Acknowledgement: The authors, wish to expressed their gratitude to members and staff of Elf, Nigeria Ltd. at Obagi for the assistance in carrying out this investigations. Special thanks goes to Ese Imuere who under take this investigation as part of her B.Sc work during her undergraduate programme with the University of Port Harcourt, Physics Department. Our sincere thanks are also extended to Prof. I.O. Owate for his suggestion during the analysis of the corrosion rate and for the development of an earlier article on this subject.

\section{REFERENCES}

Abbey, T.M. Alagoa K.D. and Nyeke K.N.O. (2003), "Mathematical Simulation of Stack Effluent Pollutant dispersion in a windy Atmosphere", J. Nig. Envir. Soc. 1 (2), 163172.

Abbey, T.M. Amakiri, J.O. and Bestman, A.R. (1997), "Effect of Pollutant Oscillation on Oil Spreading on the Sea". Int. J. Energy Research. 21, 655-660.

Akpan, A.J., LETCHA S.S. and MUSA O. (1992); "Strength Assessment of Concrete subjected to a predominantly acid Media". Proceeding of NICA, pp 9-16.

Allen, M.D. and Hilbert, G.G.A. (1992), "Specialized Surveys on buried pipelines in Nigeria", J.NICA, 99 (15) 23-29.. 
Amens, W.F. (1988), "Analysis of Mathematical Models for Pollutant Transport and Dissipation", Computer Matt. Application, 16 (10/11), 939.

Bansal, M.K. (1976); "Dispersion Model of an Instantaneous Source of Pollution in Natural streams and its Applicability to Big Blue Rivers", Environmental Modeling and Simulation Conference Proceeding; BPA 600 19-76-016, 335.

Brikby I. Harrison P. and Stevens R. (1999), “The effect of surface Transformation due to Degradation", J. European Soc. 5 (1), 37-46.

Chinemerem, I.J. (1989); "Effects of Environmental Degradation on the Electrical Characteristics of Semi-Conductors (Silicon Wafer)", B.Sc. Thesis, Department of Physics, Uniport, Nigeria.

Cleary, R.W. (1976), "Unsteady-State, Multidimensional Analytic Modeling of water Quality In Rivers", Environmental Modeling and Simulation Conference Proceeding, BPA 600/9-76-016, 434.

Cookey, R.G. and Okongwu, E.A. (1992); “A Preliminary Study of the Surges in Pipe-ToSoil Potential (Due to Telluric and other effects) on KM 278-Makurdi Section of PHMakurdi Product Pipeline". Proceedings of NICA, pp30-46.

Darry P. and Spear K. (1992), "Corrosion of Materials in $\mathrm{N}_{2}-\mathrm{H}-\mathrm{Co}$ ", J. Corrosion Science, 75 (12) 3257-77.

Dawson, J.L. and Turner, N.E.D (1988), "Corrosion Monitoring and Control", J. Inst. of Metals 4, 218-222.

Ebiogwu C.A. (1992), "Corrosion and Protection of offshore oil Production Installations" Proc. NICA, 100-103.
Ezeh, J.C. (1992); "Corrosion Control in Reinforced Concrete". Proceedings of NICA, pp.2-8.

Jain, M.K. Iyengar, S.R.K. and Jain, R.K. (2004), Numerical Methods for Scientific and Engineering Computation, New Age Int. Publisher, New Delhi, pp. 217-327.

Johnson L.B. (1992), "Current initiative on Facilities integrity management in the oil Industry", Proc. NICA, 104-110.

Mckelliget, J.W. Cross M. and Gibson R.D. (1982), "A Turbulent Fluid Flow Model of Gas Agitated Applied Math. Modeling, 6, 469-80.

Nelson, P. and Stile, J.R. (1988), "Metallurgical Failures of Offshore oil Installation", J. Inst. of Metals 4, 559-564.

Owate, I.O. (1992); "Corrosion Resistance Characteristics of Alimina and Aluminum Nitride in Hostile Industrial Environment". Proceedings of NICA, pp. 136-143.

Owate, I.O. Abbey, T.M. and Imuere E.J. (1992) "The Influence of Acidic Medium on some Electrical Properties of Copper Wire", J.NICA, 99 (15), 122-130.

Owate, I.O. and Akpata, A.N.O. (1998); "High Temperature Corrosion and Microstructural Changes of an Alumina Ceramic under Chemical Treatment". Proceedings of NICA, pp. 68-72.

Triki, E.; Hachani, L. and Raharinaivo, A. (1992); "Electrochemical Study of the Corrosion of Steel Embedded in Concretes Deteriorated by Chlorides". Proceedings of NICA, pp. 17-29.

Wenk, W.J. and Fochtman, E.G. (1972), "Mathematical Model for Flake of Pollutants in Near-shore waters", Environmental Sci. and Tech. 6 (4), 331-33 7. 